\title{
EXTRAÇÃO E PURIFICAÇÃO DE CLOROFILA A, DA ALGA Spirulina maxima: UM EXPERIMENTO PARA OS CURSOS DE QUÍMICA
}

\author{
Ana Paula Jecks Maestrin, Cláudio Roberto Neri, Kleber Thiago de Oliveira, Osvaldo Antonio Serra e Yassuko Iamamoto* \\ Departamento de Química, Faculdade de Filosofia, Ciências e Letras de Ribeirão Preto,- Universidade de São Paulo,14040-901 \\ Ribeirão Preto - SP, Brasil
}

Recebido em 30/9/08; aceito em 4/2/09; publicado na web em 3/7/09

\begin{abstract}
EXTRACTION AND PURIFICATION OF CHLOROPHYLL A ISOLATED FROM Spirulina maxima: AN EXPERIMENT FOR THE CHEMISTRY COURSES. This work describes a simple and economical experiment for the extraction and purification of chlorophyll $a$ from Spirulina maxima. Extraction and purification of natural compounds can be considered one of the most illustrative experiments that can be performed in Organic Chemistry courses. Particularly, the chromatography of dyes and pigments allows students to have a better comprehension of the chromatography separations. These compounds represent an important class of organic pigments applied in pharmaceutical, cosmetic, detergent compositions, and various other fields and can be extracted from plants and algae. To extract, separate and purify chlorophyll $a$ from associated pigments such as xanthophylls, carotenes, and pheophytins, very costly processes are reported. The present approach is perfectly adequate for use in Chemistry experiments for undergraduate students.
\end{abstract}

Keywords: chlorophyll $a$; extraction and purification; Spirulina maxima.

\section{INTRODUÇÃO}

As clorofilas destacam-se dentre os mais conhecidos pigmentos vegetais sendo responsáveis pela captura de luz solar, produção de oxigênio e açúcares através da fotossíntese.$^{1,2} \mathrm{~A}$ intensa cor verde das clorofilas deve-se a suas fortes absorções nas regiões do azul e vermelho do espectro eletromagnético, fazendo com que transmitam na região do verde. ${ }^{2}$ Presentes em quase todos os tipos de plantas, algas e algumas bactérias, estes pigmentos têm sido apontados como excelentes fotossensibilizadores, ${ }^{3}$ antioxidantes e como agentes terapêuticos no combate de diversas doenças. ${ }^{1}$

A clorofila $a$ pode ser considerada como o pigmento mais recorrente desta classe de compostos ( $75 \%$ dos pigmentos verdes presentes nas plantas), sendo empregada em várias composições farmacêuticas, como cosméticos, materiais de higiene bucal, em dietas, culinária e até mesmo em alguns detergentes. ${ }^{1}$ Muitos dos seus derivados, como a clorina e ${ }_{6}$ e o "pheophorbide", ao lado de similares sintéticos, têm sido investigados como agentes sensibilizadores na terapia fotodinâmica, ${ }^{4}$ em particular nos tratamentos de câncer de pele.

As clorofilas $a$ e $b$ (Figura 1) são encontradas quase sempre em conjunto, sendo a clorofila $a$ mais abundante na maioria dos casos. Particularmente nas algas Spirulina pacifica ou S. maxima encontrase apenas a clorofila $a$ com rendimento de até $2 \%$ do seu peso. ${ }^{1,5} \mathrm{~A}$ fórmula molecular da clorofila $a$ é dada por $\mathrm{C}_{55} \mathrm{H}_{72} \mathrm{O}_{5} \mathrm{~N}_{4} \mathrm{Mg}$ e difere da clorofila $b$ pela presença de um grupo funcional aldeído ao invés de um grupo metila no átomo de carbono 7 (Figura 1).

Historicamente, a estrutura da clorofila $a$ só foi completamente elucidada em 1960, quando Woodward confirmou a estrutura proposta para este composto através da sua síntese total. ${ }^{6}$ Pouco tempo depois Fleming ${ }^{7}$ determinou a configuração absoluta da clorofila $a$ não deixando dúvidas quanto a quaisquer aspectos estereoquímicos e, ao mesmo tempo, contribuindo para uma série de estudos no que diz respeito a este tipo de pigmento. ${ }^{8}$

Os métodos descritos na literatura para a extração/purificação da clorofila $a$ proveniente de plantas e algas verdes podem ser

*e-mail: iamamoto@ffclrp.usp.br
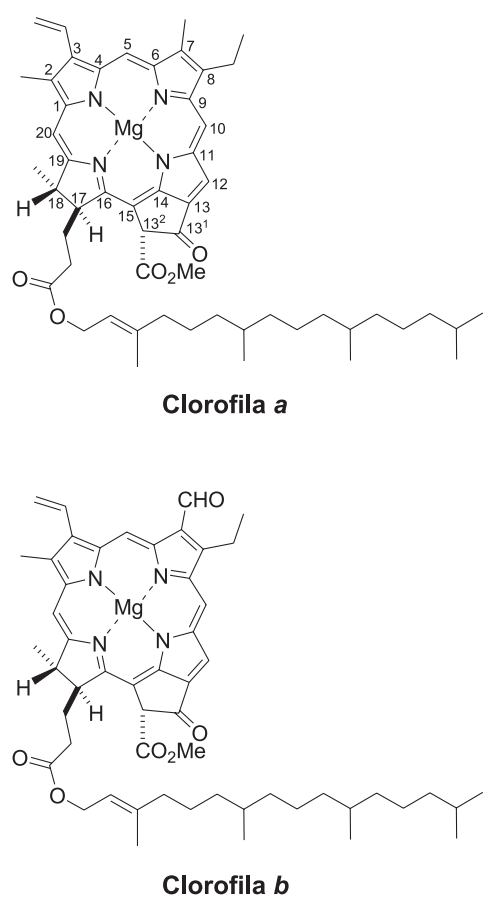

Figura 1. Estruturas das clorofilas a e $b$

considerados como processos complicados e, em alguns casos, ineficientes e dispendiosos. ${ }^{9,10}$ Geralmente a clorofila $a$ é extraída na presença de solventes como acetona ou metanol, resfriada com nitrogênio líquido e posteriormente refluxada e filtrada para sua separação do material de partida. O processo de refluxo sob atmosfera de nitrogênio e posterior filtração é repetido por várias vezes. Os pigmentos obtidos nestes processos são purificados em coluna cromatográfica de polietileno e após decantar, separar e eliminar os solventes, as frações obtidas são repurificadas em colunas cromatográficas de açúcar e finalmente cristalizadas na presença de iso-octano. ${ }^{10}$ 
Neste trabalho, relatamos um experimento econômico, eficiente e extremamente didático para extração e purificação da clorofila $a$, a partir da Spirulina maxima. Este experimento permitirá aos estudantes utilizar técnicas de extração de produtos naturais, realizar purificações cromatográficas, bem como a identificação dos mesmos por espectros UV-Vis. Tais conteúdos estão presentes em disciplinas de Química em vários cursos de graduação, sendo que a Spirulina maxima foi escolhida com o objetivo de evitar os tediosos processos de separações cromatográficas dos variados tipos de clorofila $(a, b, c 1, c 2$ e $d){ }^{2}$

\section{MATERIAIS E REAGENTES}

Os solventes utilizados foram de procedência nacional (PA). A alga Spirulina maxima pode ser adquirida em quaisquer casas de comércio de produtos naturais, fitoterápicos ou de distribuição de insumos para farmácias de manipulação. Para realização do experimento é necessário o uso de chapas de aquecimento e agitação magnética, barras magnéticas, celas de quartzo ou vidro para espectroscopia UV-Vis de $1 \mathrm{~cm}$ de caminho óptico, espectrofotômetro, evaporador rotatório e vidrarias comuns como erlenmeyer, balões de fundo redondo, funil de vidro, funil de decantação e pipetas.

\section{PLANEJAMENTO DA AULA}

A primeira etapa pode ser realizada em um período de aproximadamente $3 \mathrm{~h}$, incluindo as extrações, filtração e evaporação do extrato. Neste caso o extrato deve ser armazenado em balão fechado e protegido da luz para que num experimento posterior possa ser purificado. Para etapa de purificação são necessárias pelo menos 4 $\mathrm{h}$, incluindo o empacotamento da coluna e eluição das frações. Um tempo posterior de $3 \mathrm{~h}$ deverá ser utilizado para remoção do metal $\left(\mathrm{Mg}^{2+}\right)$ lembrando que, até esta etapa, se deve manter o extrato seco e protegido da luz (recomenda-se cobrir o balão com papel alumínio e armazenar em armário fechado).

\section{PROCEDIMENTO DE EXTRAÇÃO}

Foram adicionados $5 \mathrm{~g}$ de Spirulina maxima a um erlenmeyer de $250 \mathrm{~mL}$, misturando-se em seguida com $50 \mathrm{~mL}$ de $\mathrm{CH}_{2} \mathrm{Cl}_{2}$ à temperatura ambiente. A suspensão foi agitada por 20 min e posteriormente filtrada em papel de filtro comum (dobra pregueada), reservando-se o filtrado. O resíduo sólido foi então submetido ao mesmo tratamento com $\mathrm{CH}_{2} \mathrm{Cl}_{2}$ por mais 3 vezes. Os extratos obtidos foram reunidos e concentrados em um evaporador rotatório sob pressão reduzida até o volume final de $5 \mathrm{~mL}$ (aproximadamente). Neste caso, além da clorofila $a$, outros pigmentos como $\beta$-caroteno e xantofilas são extraídos. Para efeito de comparação com os produtos a serem posteriormente purificados, realizou-se uma análise de UV-Vis deste extrato, diluindo-se 1 gota do extrato em $4 \mathrm{~mL}$ de $\mathrm{CH}_{2} \mathrm{Cl}_{2}$. A linha de base (branco) do aparelho de UV-Vis foi feita com $\mathrm{CH}_{2} \mathrm{Cl}_{2}$ (Figura 2).

\section{PURIFICAÇÃO DO EXTRATO E ANÁLISE DOS PRODUTOS}

A purificação do extrato foi realizada em uma coluna cromatográfica de sílica gel (200-400 mesh, $2 \mathrm{~cm}$ de diâmetro e $15 \mathrm{~cm}$ de altura) empacotada com tolueno em uma capela de exaustão. A presença de luz no ambiente onde é realizada a cromatografia pode induzir a degradação parcial da clorofila $a$, entretanto, o tempo gasto para o processo (4 h) é relativamente curto, não implicando em grandes perdas. Recomenda-se apenas a não utilização da iluminação interna da capela de exaustão durante a eluição. A primeira eluição foi realizada com o mesmo solvente de empacotamento da coluna (tolueno) para

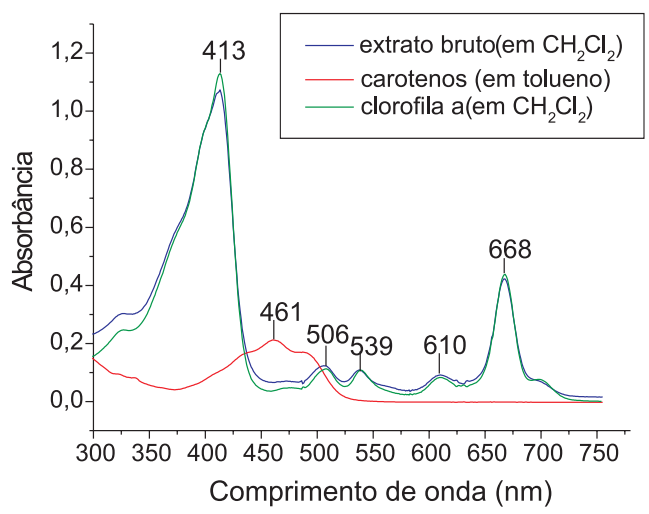

Figura 2. Espectros de UV-Vis do extrato bruto, da fração de caroteno e da clorofila a

remover as bandas amarela e laranja, ricas em caroteno e xantofila (Figura 3); nesta etapa são necessários pouco mais de $150 \mathrm{~mL}$ de tolueno. Posteriormente eluiu-se com $\mathrm{CH}_{2} \mathrm{Cl}_{2}$ para se obter a grande fração verde/azulada correspondente à clorofila $a$ (Figura 3). Esta terceira fração (rica em clorofila $a, \mathrm{C}_{55} \mathrm{H}_{72} \mathrm{O}_{5} \mathrm{~N}_{4} \mathrm{Mg}$ ) foi caracterizada por espectroscopia UV-Vis em $\mathrm{CH}_{2} \mathrm{Cl}_{2}$, (Figura 2, $\lambda_{\text {max }}^{\mathrm{CH}_{2} \mathrm{Cl}_{2}}$ (nm): 413 (banda Soret), 506, 539, 610 e 668) diluindo-se 1-2 gotas da fração coletada em $4 \mathrm{~mL}$ de $\mathrm{CH}_{2} \mathrm{Cl}_{2}$. Em seguida, toda a fração contendo a clorofila $a$ foi concentrada removendo-se o solvente sob pressão reduzida e a amostra obtida foi armazenada sob o abrigo da luz para evitar processos de auto-oxidação. Também se realizou uma análise por espectrofotometria na região do UV-Vis da fração eluída em tolueno (rica em carotenos e xantofila) ajustando-se a linha de base do aparelho com tolueno (Figura 2). A superposição dos espectros na região do UV-Vis do extrato bruto, carotenos e clorofila $a$ traz um bom indicativo da composição do extrato. (a)

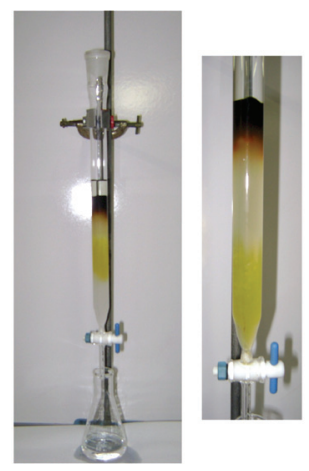

(b)


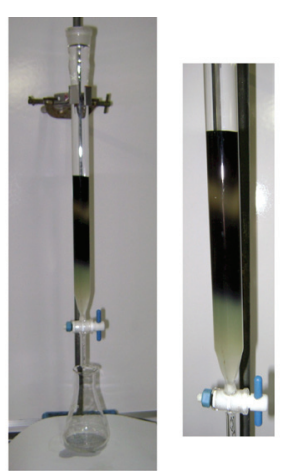

Figura 3. Procedimento de purificação da clorofila a por cromatografia em coluna 
Como pode ser visto na Figura 2, o perfil do espectro na região do UV-Vis do extrato é praticamente igual ao da clorofila $a$ isolada, mostrando que, no extrato, este pigmento pode ser encontrado majoritariamente. Cabe salientar que os coeficientes de absortividade molar das clorofilas e dos carotenos são da mesma ordem de grandeza (1-1,5 x $\left.10^{5} \mathrm{~L} \mathrm{~mol}^{-1} \mathrm{~cm}^{-1}\right)^{6}$ confirmando que a presença de carotenos no extrato é de fato pequena e sequer pode ser detectada pelo espectro na região do UV-Vis do extrato bruto. Deve-se destacar ainda dois dados importantes de identificação da clorofila $a$. O primeiro é a presença da principal banda de absorção de um derivado do tipo porfirina/clorina (banda Soret) encontrada geralmente entre $400 \mathrm{e}$ $420 \mathrm{~nm}$. A segunda banda importante a ser identificada é a banda em $668 \mathrm{~nm}$, relativamente intensa e típica de derivados de clorofila (banda Q mais intensa). Estes dois dados devem ser utilizados como critério de caracterização neste experimento.

O rendimento final da clorofila $a$ foi de $1,0 \%(50 \mathrm{mg})$ considerando a massa inicial de Spirulina maxima ( $5 \mathrm{~g})$. Note que este é um experimento adaptado para condições de uma aula experimental, sendo que, utilizando-se quantidades maiores de alga e tempos de extração em torno de $1 \mathrm{~h}$, o rendimento pode chegar a $2 \%$ em massa. ${ }^{1}$

\section{DESMETALAÇÃO DA CLOROFILA $A$}

A clorofila $a$ pode, ainda, ser estocada na sua forma livre (sem $\mathrm{Mg}^{2+}$ ), o que evita os processos de fotodegradação. Para isso a clorofila $a$ foi tratada com $5 \mathrm{~mL}$ de uma solução etanólica $(\sim 1 \%)$ de ácido acético por $2 \mathrm{~h}$ (Esquema 1). O solvente foi evaporado sob pressão reduzida e o resíduo foi diluído com $50 \mathrm{~mL}$ de $\mathrm{CH}_{2} \mathrm{Cl}_{2}$. A mistura foi extraída duas vezes com porções de $50 \mathrm{~mL}$ de água utilizando-se um funil de decantação e a fase orgânica foi então separada, secada com cerca de $5 \mathrm{~g}$ de sulfato de sódio anidro, filtrada e o solvente removido sob pressão reduzida (evaporador rotatório), obtendo-se o produto denominado feofitina $a$, que foi cristalizado em $1 \mathrm{~mL}$ de metanol. Fórmula molecular: $\mathrm{C}_{55} \mathrm{H}_{74} \mathrm{O}_{5} \mathrm{~N}_{4}$, características espectrais UV-Vis (nm): 412 (banda Soret), 507, 540, 610 e 667 - Figura 4). Experimentos de espectrometria de massas, e RMN de ${ }^{1} \mathrm{H}$ podem ser executados complementando ainda mais a aula.

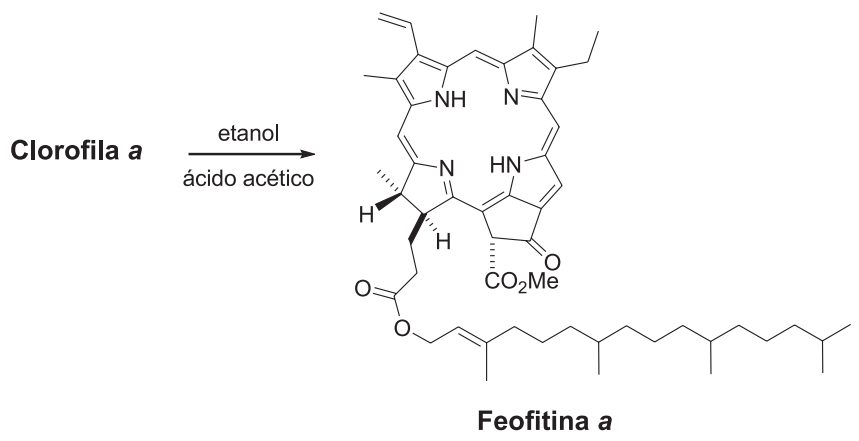

Esquema 1. Remoção do $\mathrm{Mg}^{2+}$ da clorofila

\section{CONCLUSÕES}

Por envolver processos básicos de extração de produtos naturais, técnicas de separação cromatográfica e identificação de compostos heterocíclicos naturais que apresentam relevância como pigmentos da fotossíntese, este experimento é adequado e consiste numa proposta simples, de baixo custo e altamente didática para disciplinas introdutórias à química experimental em cursos de graduação. Além disso, a possível abordagem de aspectos de elucidação estrutural envolvendo técnicas espectroscópicas e/ou espectrométricas permite que seja apli-

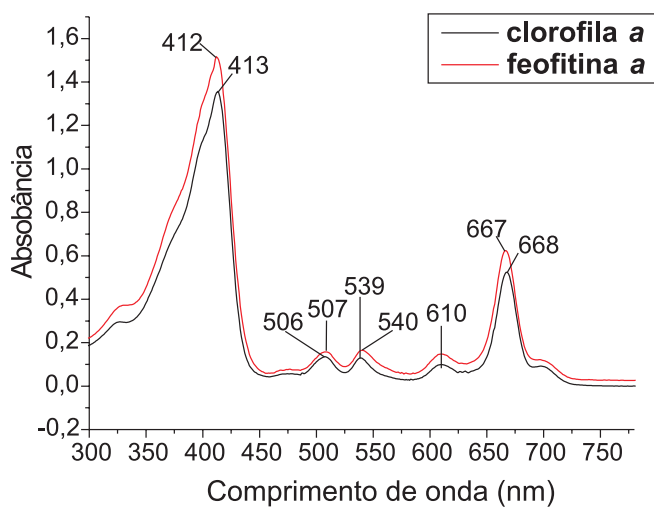

Figura 4. Espectros de UV-Vis da clorofila a e da feofitina a

cado em cursos experimentais de química orgânica mais avançados.

Esta prática permite a utilização de alguns conceitos de Química, que são essenciais à formação dos estudantes, em um tempo curto de aula (em torno de $10 \mathrm{~h}$ ).

\section{AGRADECIMENTOS}

À FAPESP, CNPq e CAPES pelo apoio financeiro. C. R. Neri, Y. Iamamoto e O. A. Serra agradecem ao CNPq pelos auxílios, 501024/2008-5, 475961/2006-4 e 470616/2007-5, respectivamente. K. T. de Oliveira agradece à FAPESP pela bolsa de Pós-Doc (proc. 07/03589-4). Agradecemos também a P. C. de Sousa Filho pelas valiosas sugestões neste trabalho quanto à sua adaptação para os padrões didáticos.

\section{REFERENCIAS}

1. Ma, L.; Dolphin, D.; Phytochemistry 1999, 50, 195.

2. WIKIPÉDIA. Desenvolvido pela Wikimedia Foundation. Apresenta conteúdo enciclopédico. Disponível em: http://pt.wikipedia.org/w/index. php?title=Clorofila\&oldid=12306401, acessada em Setembro 2008.

3. Lee, S. H.,; Jagerovic, N.; Smith, K. M.; J. Chem. Soc. Perkin. Trans. 1993, 1, 2369; Pandey, R. K.; Zheng, G. Em The Porphyrin Handbook; Kadish, K. M.; Smith, K. M.; Guilard R., eds.; Academic Press: USA, 2000, vol. 6, cap. 43; Neri, C. R.; Maestrin-Prado, A. P. J.; Ribeiro, A. O.; Serra, O. A.; Iamamoto, Y.; Mater. Sci. 2002, 2, 97.

4. de Oliveira, K. T.; Silva, A. M. S.; Tomé, A. C.; Neves, M. G. P. M. S.; Neri, C. R.; Garcia, V. S.; Serra, O. A.; Iamamoto, Y.; Cavaleiro, J. A. S.; Tetrahedron 2008, 64, 8709.

5. Chronakis, I. S.; Galatanu, A. N.; Nylander T.; Lindman B.; Colloids Surf., A 2000, 173, 181.

6. Woodward, R. B.; Ayer, W. A.; Beaton, J. M.; Bickelhaupt, F.; Bonnett, R.; Buchschacher, P.; Closs, G. L.; Dutler, H.; Hannah, J.; Hauck, F. P.; Itô, S.; Langemann, A.; Le Goff, E.; Leimgruber, W.; Lwowski, W.; Sauer, J.; Valenta, Z.; Volz H.; J. Am. Chem. Soc. 1960, 82, 3800.

7. Fleming, I.; Nature 1967, 216,151.

8. Woodward, R. B.; Ayer, W. A.; Beaton, J. M.; Bickelhaupt F.; Bonnett, R.; Buchschacher, P.; Closs, G. L.; Dutler, H.; Hannah, J.; Hauck, F. P.; Itô, S.; Langemann, A.; Le Goff, E.; Leimgruber, W.; Lwowski, W.; Sauer, J.; Valenta, Z.; Volz, H.; Tetrahedron 1990, 46, 7599

9. Smith, K. M.; Goff, D. A.; Simpson, D. J.; J. Am. Chem. Soc. 1985, 107, 4946.

10. Anderson, A. F. H.; Calvin, M.; US pat. 3,258,467 1966; Pandey, R. K.; Dougherty, T. J.; US pat. 5,093,349 1992; Smith, K. M.; Lee, S. J. H.; US pat. 5,330,741 1994. 